\title{
Fatores de risco associados à Infecção do Trato Urinário (ITU) em mulheres: uma revisão integrativa de literatura
}

\author{
Risk factors associated with Urinary Tract Infection (UTI) in women: an integrative literature \\ review
}

Factores de riesgo asociados con la Infección del Tracto Urinario (ITU) en mujeres: una revisión integradora de la literatura

Clarisse Queiroz Lima de Araújo ORCID: https://orcid.org/0000-0001-6202-7530 Centro Universitário de Patos de Minas, Brasil E-mail: clarisselima@unipam.edu.br

Annelize Florêncio Rabelo ORCID: https://orcid.org/0000-0003-2689-7824 Centro Universitário de Patos de Minas, Brasil E-mail: annelizerabelo@unipam.edu.br

Bárbara Queiroz de Figueiredo ORCID: https://orcid.org/0000-0003-1630-4597 Centro Universitário de Patos de Minas, Brasil

E-mail: barbarafigueiredo@unipam.edu.br

Bethânya Helena Silva de Oliveira ORCID: https://orcid.org/0000-0001-7284-6046 Centro Universitário de Patos de Minas, Brasil E-mail: bethanyahso@unipam.edu.br

Maria Laura Alves Freitas

ORCID: https://orcid.org/0000-0002-7167-1608 Centro Universitário de Patos de Minas, Brasil E-mail: marialauraaf@unipam.edu.br

Rúbia Carla Oliveira

ORCID: https://orcid.org/0000-0002-7162-3497 Centro Universitário de Patos de Minas, Brasil E-mail: rubiacoliveira@ unipam.edu.br

\begin{abstract}
Resumo
Introdução: o sistema urinário é responsável por remover os produtos finais do metabolismo e regular os fluidos corporais. As infecções do trato urinário (ITU) são queixas recorrentes, acometem principalmente mulheres e tem como fatores de risco questões anatômicas, má higiene, idade, relações sexuais e hábitos de vida. Objetivo: evidenciar quais são os principais fatores de risco relacionados às infecções do trato urinário em mulheres. Metodologia: Trata-se de uma pesquisa descritiva do tipo revisão integrativa da literatura para identificar os fatores de risco para infecções do trato urinário em mulheres. A pesquisa foi online nas diversas bases de dados como PubMed MEDLINE, Scielo, CDSR, Google Scholar, BVS e EBSCO. O principal critério de inclusão foi o período 2015-2021 e com os descritores foram encontrados 23 artigos. Resultados e discussão: A infecção do trato urinário (ITU) é a invasão de microorganismos no sistema urinário, que causa uma inflamação local. Dentre as principais características da maior incidência em mulheres estão os fatores anatômicos como a proximidade do trato urinário com a região perianal e o comprimento da uretra. Outros fatores estão relacionados a hormônios, a hábitos higiênicos evacuatórios e sexuais e a aspectos ambientais. Conclusão: Portanto, as mulheres realmente apresentam maior prevalência, por isso se faz importante a adoção de medidas preventivas e de ações educativas, a fim de garantir a modificação comportamental profilática, incluindo higiene, ingestão hídrica e alimentação, a fim de evitar os impactos e a consequente redução na qualidade.
\end{abstract}

Palavras-chave: Infecção urinária; Prevalência; Fatores de risco; Mulheres; Sistema urinário.

\begin{abstract}
Introduction: the urinary system is responsible for removing the end products of metabolism and regulating body fluids. Urinary tract infections (UTI) are recurrent complaints, mainly affecting women and their risk factors are anatomical issues, poor hygiene, age, sexual relations and lifestyle habits. Objective: to show the main risk factors related to urinary tract infections in women. Methodology: This is a descriptive research of the integrative literature
\end{abstract}


review type to identify the risk factors for urinary tract infections in women. The search was online in several databases such as PubMed MEDLINE, Scielo, CDSR, Google Scholar, BVS and EBSCO. The main inclusion criterion was the period 2015-2021 and with the descriptors 23 articles were found. Results and discussion: Urinary tract infection (UTI) is the invasion of microorganisms in the urinary system, which causes local inflammation. Among the main characteristics of the higher incidence in women are anatomical factors such as the proximity of the urinary tract to the perianal region and the length of the urethra. Other factors are related to hormones, hygienic evacuation and sexual habits, and environmental aspects. Conclusion: Therefore, women do have a higher prevalence, which is why it is important to adopt preventive measures and educational actions in order to ensure prophylactic behavioral modification, including hygiene, water intake and food, in order to avoid impacts and the consequent reduction in quality.

Keywords: Urinary infection; Prevalence; Risk factors; Women; Urinary system.

\begin{abstract}
Resumen
Introducción: el sistema urinario se encarga de eliminar los productos finales del metabolismo y regular los fluidos corporales. Las infecciones del tracto urinario (ITU) son molestias recurrentes, que afectan principalmente a las mujeres y sus factores de riesgo son problemas anatómicos, mala higiene, edad, relaciones sexuales y hábitos de vida. Objetivo: mostrar los principales factores de riesgo relacionados con las infecciones del tracto urinario en la mujer. Metodología: Se trata de una investigación descriptiva del tipo revisión integradora de la literatura para identificar los factores de riesgo de infecciones del tracto urinario en mujeres. La búsqueda se realizó en línea en varias bases de datos como PubMed MEDLINE, Scielo, CDSR, Google Scholar, BVS y EBSCO. El principal criterio de inclusión fue el período 2015-2021 y con los descriptores se encontraron 23 artículos. Resultados y discusión: La infección del tracto urinario (ITU) es la invasión de microorganismos en el sistema urinario, que causa inflamación local. Entre las principales características de mayor incidencia en mujeres se encuentran factores anatómicos como la proximidad del tracto urinario a la región perianal y la longitud de la uretra. Otros factores están relacionados con las hormonas, la evacuación higiénica y los hábitos sexuales y los aspectos ambientales. Conclusión: Por tanto, las mujeres sí tienen una mayor prevalencia, por lo que es importante adoptar medidas preventivas y acciones educativas para asegurar la modificación profiláctica de la conducta, incluyendo la higiene, la ingesta de agua y la alimentación, con el fin de evitar impactos y la consecuente reducción de calidad.
\end{abstract}

Palabras clave: Infección urinaria; Predominio; Factores de riesgo; Mujeres; Sistema urinario.

\title{
1. Introdução
}

O sistema urinário é composto por dois rins, ureteres, bexiga urinária e uretra. Esse conjunto de órgãos é responsável por remover os produtos finais do metabolismo e por regular fluidos corporais por meio da filtração glomerular. Dessa forma, o sistema atua removendo resíduos e minerais indesejáveis do sangue, os quais podem causar toxidade ao corpo quando não forem eliminados de forma adequada. Junto ao excesso de água do corpo, esses resíduos são expelidos em forma de urina, regulando a composição química do sangue (Santos et al. 2019).

Atrás apenas das infecções respiratórias, a infecção do trato urinário (ITU) é uma das principais causas de consultas médicas e caracteriza-se pela proliferação de microrganismos patógenos no trato urinário (Fioravante et al., 2017). As infecções podem ser sintomáticas ou assintomáticas e podem envolver o trato urinário baixo (uretra e bexiga), como pielonefrite, e/ou trato urinário alto (rins e ureteres), como a cistite e uretrite (Vaz et al., 2020). De acordo com Oliveira et al. (2021), o diagnóstico quantitativo da ITU é representado pelo crescimento bacteriano acima de 100.000 unidades formadoras de colônia por mililitro (ml) de urina (100.000 UFC/mL). Quando a ITU é sintomática, entre os principais sintomas clínicos, destaca-se a polaciúria, disúria, dor lombar, urgência miccional, febre, alteração de cor e odor da urina. A infecção inicia-se com a inflamação da uretra e, caso não ocorra o tratamento adequado, essa inflamação pode atingir a bexiga, os ureteres e os rins, sendo que os casos mais graves podem evoluir para a septicemia e óbito (Anacleto et al. 2016).

As ITUs acometem, predominantemente, três faixas etárias. O primeiro grupo contempla crianças com até 6 anos de idade, principalmente recém nascidas do sexo masculino, o que pode estar relacionado a anormalidades anatômicas ou funcionais do aparelho urinário. O segundo abarca jovens, majoritariamente mulheres, uma vez que no sexo feminino a abertura da uretra encontra-se anterior às aberturas da vagina e do ânus, sendo que essa extensão mais curta da uretra das mulheres e a sua proximidade com a região perianal faz com que elas sejam mais propensas a desenvolver infecções 
bacterianas no trato urinário. E o terceiro engloba idosos acima de 60 anos, frequentemente relacionado a alterações morfológicas e funcionais da bexiga e a hospitalização com ou sem o uso de sonda vesical (Machado et al., 2019).

Além do sexo e da faixa etária, fatores como hábitos de vida, higiene inadequada, período gestacional, menopausa e frequência das relações sexuais também podem ser fatores de risco para o desenvolvimento da ITU (Santos et al., 2018). Sob essa perspectiva, o objetivo desse estudo é evidenciar quais são os principais fatores de risco relacionados às infecções do trato urinário em mulheres.

\section{Metodologia}

Trata-se de uma pesquisa descritiva do tipo revisão integrativa da literatura, que buscou responder quais são os fatores de risco para ocorrência de infecções do trato urinário em mulheres. A pesquisa foi realizada através do acesso online nas bases de dados National Library of Medicine (PubMed MEDLINE), Scientific Electronic Library Online (Scielo), Cochrane Database of Systematic Reviews (CDSR), Google Scholar, Biblioteca Virtual em Saúde (BVS) e EBSCO Information Services, no mês de agosto de 2021. Para a busca das obras, foram utilizadas as palavras-chaves presentes nos descritores em Ciências da Saúde (DeCS): em inglês - "urinary tract infection", "risk factors", "women", "urinary tract infections"; em português - "infecção do trato urinário", "fatores de risco", "mulheres", "infecções urinárias"; e em espanhol - "infección del tracto urinario", "factores de riesgo", "mujeres", "infecciones del tracto urinario".

Como critérios de inclusão, foram considerados artigos originais, que abordassem o tema pesquisado e que permitissem acesso integral ao conteúdo do estudo, publicados no período de 2015 a 2021, em inglês, português e espanhol. O critério de exclusão foi imposto naqueles trabalhos que não estavam em inglês, português e espanhol, que não tinham passado por processo de Peer-View e que não abordassem os fatores de risco para ocorrência de infecções do trato urinário em mulheres. A estratégia de seleção dos artigos seguiu as seguintes etapas: (i) busca nas bases de dados selecionadas; (ii) leitura dos títulos de todos os artigos encontrados e exclusão daqueles que não abordavam o assunto; (iii) leitura crítica dos resumos dos artigos; e (iv) leitura na íntegra dos artigos selecionados nas etapas anteriores. Assim, totalizaram-se 23 artigos científicos para a revisão integrativa da literatura, com os descritores apresentados acima, dos últimos seis anos e em línguas portuguesa, inglesa e espanhola.

\section{Resultados e Discussão}

A infecção do trato urinário (ITU) é definida pela invasão, colonização e multiplicação de microorganismos no sistema urinário, o que causa uma inflamação local (Fioravante et al., 2017). Tal quadro pode manifestar-se tanto de forma assintomática, quanto sintomática. Em relação à sintomatologia, trata-se de um panorama clínico extremamente característico, o que favorece o autodiagnostico de muitas mulheres. Esses sintomas comumente consistem em disúria, polaciúria, poliúria, anúria, noctúria e oligúria, todavia, em casos de maior comprometimento, outros sinais podem estar presentes (Paula et al., 2016).

Ainda que o trato urinário seja considerado estéril, estima-se que a maioria das ITUs ocorra em função de contaminações por bactérias gram-negativas. Nesse âmbito, o principal patógeno responsável por desencadear esse quadro é a Escherichia coli, representando cerca de $80 \%$ de todos os episódios (Vaz et al., 2020). Além dos casos de origem bacteriana, o contágio pode dar-se por fungos, vírus e parasitas, que acometem, majoritariamente, pacientes imunodeprimidos, diabéticos ou indivíduos que apresentam anormalidades anatômicas (Anacleto et al., 2016). Essa contaminação do sistema urinário pode ocorrer de três maneiras distintas. A principal, é advinda da via ascendente, a partir da flora fecal e uretral. Outra maneira, é pela via hematogênica, na qual a bactéria infecta o sangue e, secundariamente, contamina o aparelho urinário. Por fim, pode se dar por meio da via linfática, sendo uma promotora da disseminação infeciosa pelos vasos linfáticos (Oliveira et al., 2021). 
Indubitavelmente, a ITU, patologia clínica multifatorial, apresenta grande incidência global. Isso torna-se nítido ao verificar que tal condição ocupa a posição de segunda infecção mais comum em seres humanos, imediatamente após as infecções do trato respiratório (Paulo et al., 2021). Nesse sentido, estima-se que 86\% dos indivíduos já tenham apresentado algum quadro de ITU ao longo da vida (Lia et al., 2015). É importante ressaltar que essa ocorrência, assim como a sua etiologia, é diretamente influenciada por fatores como sexo, idade, comorbidades e distribuição geográfica (Machado et al., 2019). Nesse contexto, identifica-se maior prevalência de ITU no sexo feminino em comparação ao sexo masculino (Castro et al., 2020). Essa predominância é resultado de múltiplos fatores, dos quais destacam-se a anatomia do sistema urogenital, alterações fisiológicas e fatores comportamentais de risco, como hábitos de higiene e atividade sexual (Santos et al., 2019).

A variação anatômica é considerada o principal fator de risco para a instauração da ITU no sexo feminino, visto que mulheres apresentam maior proximidade do trato urinário com a região perianal (Oliveira et al., 2021). Além disso, a uretra feminina é significativamente mais curta que a uretra masculina. Essas características anatomofisiológicas facilitam a ascensão de enterobactérias rumo ao aparelho urinário. Sendo assim, a distância entre o ânus e a uretra é inversamente proporcional ao risco de desenvolvimento de uma ITU (Alves et al. 2018).

De maneira análoga, gestantes apresentam maiores riscos de desenvolverem quadros de ITU em decorrência de mudanças fisiológicas que ocorrem nesse período (Estrela et al. 2019). Os rins diminuem a sua capacidade máxima de concentrar urina durante o período gestacional, fato que reduz a atividade antibacteriana e restringe a excreção de potássio, ao passo que amplia a excreção de glicose e aminoácidos. Assim, esse meio torna-se favorável para a proliferação bacteriana (Santos et al., 2018). Outro fator considerável refere-se às alterações hormonais, especialmente relacionadas à progesterona, que ocasionam dilatação da pelve renal e, consequentemente, certo estreitamento dos ureteres, o que explica o retardo do débito urinário (Tavares, 2017). Além desse aspecto, o aumento da circulação sanguínea na região pélvica, durante a gravidez, ocasiona o aumento da umidade e do tamanho do útero, fatores que facilitam a grande concentração de bactérias nessa região. Todas essas alterações presentes são potencializadas em função de uma menor imunidade gestacional (Lia et al., 2015).

Outro item identificado como fator de risco para a ITU foi a questão higiênica, tanto no que se refere à eliminação intestinal quanto ao coito. Isso manifesta-se ao passo que bactérias circunvizinhas da região genital contaminam a uretra e, consequentemente, causam infecções (Paulo et al., 2021). Nesse sentido, após as evacuações, o ideal é que a higiene íntima seja realizada com o papel higiênico no sentido ântero-posterior ou com água e sabão. Entretanto, o excesso de limpeza em mulheres, possivelmente, torna-se prejudicial, dado que pode alterar o $\mathrm{pH}$ da área genital, favorecendo a invasão bacteriana no trato urinário (Fioravante et al., 2017).

No que tange à susceptibilidade relacionada ao coito, mulheres ativas sexualmente tendem a apresentar mais episódios de ITUs comparativamente a mulheres não sexualmente ativas (Alves et al. 2018). Essa situação torna-se mais arriscada durante práticas sexuais desprotegidas. Isso verifica-se uma vez que a uretra feminina fica exposta às bactérias ou aos fungos provenientes das mucosas contaminadas dos seus parceiros sexuais. Além disso, existe o risco dessas mulheres contraírem infecções sexualmente transmissíveis (Paulo et al., 2021). Da mesma forma, o sexo anal sem o uso de preservativo pode propiciar a contaminação fecal-perineal-uretral, configurando grande fator de risco para as infecções causadas por bactérias entéricas (Paula et al., 2016).

Sob esse mesmo viés, o uso de determinados métodos contraceptivos, como o diafragma e o espermicida, também tem sido considerado preditor de ITU em mulheres sexualmente ativas. No caso específico do diafragma, pode gerar obstrução uretral. Combinado a esse método, o gel espermicida acarreta alterações no $\mathrm{pH}$ e na flora vaginal, por meio da perda de lactobacilos produtores de peróxido de hidrogênio (H2O2). Esse composto químico é um importante regulador da microbiota urogenital, responsável por manter a acidez do $\mathrm{pH}$ vaginal, $\log$, a sua ausência favorece a ascendência de bactérias ao trato 
urinário. Paralelamente a isso, o próprio sêmen ejaculado é considerado um fator capaz de alterar o $\mathrm{pH}$ vaginal e, consequentemente, a flora vaginal natural (Paula et al., 2016).

Nessa conjuntura, mulheres no período de pós-menopausa, juntamente com aquelas sexualmente ativas, formam as duas populações femininas de maior risco para tais infecções (Faria et al., 2018). Essa recorrência deve-se ao fato de que, após à interrupção da menstruação, percebe-se certa depleção hormonal, que é bastante comum nessa etapa da vida, mas que contribui para que mulheres nessa faixa etária tornem-se mais susceptíveis a infecções baixas (Arroyo et al. 2020). Sob essa lógica, o estrogênio é responsável pela restauração do glicogênio nas células das regiões vaginal e uretral, bem como por manter o pH ácido nessa área. Alterações em seus níveis causam mudanças significas, que levam a uma perda de lactobacilos, alterações na microbiota normal e no pH, e atrofia vaginal (Alves et al. 2018). Dessa maneira, a redução desse hormônio acarreta uma maior vulnerabilidade à colonização de bactérias nesse local (Fernandes et al., 2020).

Ademais, existem evidências que apontam envolvimento genético na predisposição de mulheres desenvolverem ITU de repetição. Nesse caso, tal herança estaria associada a alterações na resposta dessas hospedeiras relacionadas com a diminuição das defesas imunitárias. Isso ocorreria devido à variabilidade genética gerada por um silenciamento de interleucina (IL-8) e seus receptores em mulheres com maior tendência de desenvolver essa infecção e em seus familiares (Haddad et al. 2018). Para mais, essa patologia é mais prevalente em mulheres com parentes que apresentam história pregressa de ITU recorrente, o que reafirma essa base genética familiar (Alves et al. 2018).

Além dos fatores de risco individuais, existem também fatores de risco ambientais para ITUs em mulheres. É possível notar influência determinística da sazonalidade no aumento dessas infecções, sendo os meses de verão os mais acometidos (Simmering et al., 2017). Assim, climas mais quentes favorecem atividades aquáticas e, consequentemente, o uso prolongado de roupas de banho úmidas. Esse hábito colabora com o crescimento e com a proliferação de patógenos no trato urinário, pois esses agentes dependem da água e do calor para executarem suas funções metabólicas (Oliveira et al. 2018). Outra explicação biologicamente plausível correlaciona a elevação das temperaturas e graus inferiores de hidratação, o que resulta em níveis mais baixos de formação de urina e de eliminação de microrganismos urinários potenciais (Simmering et al., 2017).

Outro elemento a ser destacado, é quanto ao papel da hipovitaminose D como um predisponente de ITUS na população feminina, especialmente naquelas em idade reprodutiva. Tal efeito ocorre em virtude de a vitamina $\mathrm{D}$ funcionar como um potencial regulador imunológico, capaz de prevenir essas infecções. O mecanismo relacionado a essa proteção envolve a estimulação dessa vitamina sobre as células epiteliais da bexiga, que secretam e expressam a catelicidina, peptídeo antimicrobiano humano, de modo a proteger o trato urinário inferior. Para mais, essa substância também apresenta efetividade contra certos protozoários e fungos. Ainda é perceptível que a vitamina D modula o sistema imune adaptativo ao produzir citocinas das células T e B e ao suprimir processos inflamatórios (Ali et al., 2020).

Por fim, o uso de cateter também mostra certa periculosidade no sentido de propiciar ITUs no sexo feminino. Esse componente não orgânico na uretra pode estar infectado ou ser contaminado durante a própria técnica de passagem da sonda. Desse modo, o uso crônico desse instrumento ou múltiplas introduções dele favorecem a colonização do aparelho urinário por bactérias oportunistas (Paulo et al., 2021).

Consoante a isso, é imprescindível salientar que os fatores de risco para ITUs no sexo feminino podem ser potencializados por agravantes que facilitem a persistência da infecção. Como exemplos, pode-se considerar quadros de obesidade, de imunidade suprimida, de tumores ou de intervenções recentes no trato urinário (Oliveira et al., 2021). Nesse âmbito, tais agravantes contribuem para o desenvolvimento de complicações dos quadros infecciosos. Assim, destacam-se a resistência e a virulência dos microrganismos, bem como respostas imunológicas ineficazes, comorbidades ou tratamentos inadequados (Anacleto et al., n.d.) Portanto, essa discussão acerca dos fatores de risco para ITU na população feminina torna- 
se relevante, visto que a vida dessas mulheres é significativamente afetada, uma vez que há comprometimento das relações sociais, laborais, familiares e sexuais (Faria et al., 2018).

\section{Conclusão}

Em face do exposto, é evidente que o sexo feminino representa perfil determinante de prevalência e de susceptibilidade em relação à ITU. Isso se dá ao considerar que essa infecção possui caráter multifatorial, além do fato de as mulheres estarem expostas a fatores de risco consideráveis que corroboram para essa instauração. Nesse contexto, é de suma importância a adoção de medidas preventivas com o intuito de reduzir o impacto desses fatores e, consequentemente, a incidência da infecção nesse grupo. Logo, ações de educação em saúde tornam-se primordiais, visando a modificações comportamentais profiláticas, tais como hábitos miccionais coerentes com as necessidades, higiene adequada, uso de preservativos, ingestão hídrica regular e otimização imunológica por meio de alimentação saudável. Nessa perspectiva, pode-se afirmar que as ITUs são um problema de saúde pública, dado que interferem substancialmente na rotina das mulheres, causando impactos físicos, mentais, emocionais e sociais que reduzem a sua qualidade de vida.

\section{Referências}

Ali, S. B., Perdawood, D., Abdulrahman, R. M., Al Farraj, D. A., \& Alkubaisi, N. A. (2020). Vitamin D deficiency as a risk factor for urinary tract infection in women at reproductive age. Saudi Journal of Biological Sciences, 27(11), 2942-2947. https://doi.org/10.1016/j.sjbs.2020.08.008

Alves, A. R. M. S. (2018). Estratégias profiláticas da infecção urinária recorrente não complicada na mulher adulta saudável. Mestrado Integrado em medicina: Universidade de Porto, 1-38.

Anacleto, C., Apolinário, P. A., Ewillyn, T., \& Monteiro, S. (2016). Incidência de infecções do trato urinário no município de Patos, Paraíba Incidence of urinary tract infections in the municipality of Patos, 71-77.

Arroyo, J. C. L., et al. (2020). Prevalência de infecção do trato urinário entre pacientes atendidos na Unidade de Pronto Atendimento (UPA) no Município de Passos - MG. Revista Multidisciplinar e de psicologia, 15 (54), 603-616.

Castro, B. G. de, Pinto, L. S., \& Souto, R. C. F. (2020). Prevalência de bactérias Gram-positivas em infecção do trato urinário. Revista Brasileira de Análises Clínicas, 51(4), 1-6. https://doi.org/10.21877/2448-3877.201900791

dos Santos, M. J. A., Porcy, C., \& Menezes, R. A. de O. (2019). Etiologia e perfil de resistência bacteriana em uroculturas de pacientes atendidos em um hospital público de Macapá-Amapá, Brasil. Um estudo transversal TT - Etiology and profile of bacterial resistance in urine cultures of patients seen at a public hosp. Diagn. Tratamento, 24(4), [135-142]. http://fi-admin.bvsalud.org/document/view/5eue2

Estrela, Y. C. A., et al. (2019). Incidência de infecções do trato urinário no município de Patos, Paraíba. Brazilian Archives of Health and Encironment, 1 (1), 71-77.

Faria, C. A., Lourenção, L. G., Quintanilha, D. de O., Vieira, M. da S., De Andrade, P. de F. L., \& Eduardo, J. C. C. (2018). Qualidade de vida de mulheres com infecções recorrentes do trato urinário em atendimento ambulatorial. Fisioterapia Brasil, 19(3), 329-336. https://doi.org/10.33233/fb.v19i3.2064

Fernandes, G., Bald, J. C., Emanuel, A., Bonetti, D. B., \& Passolongo, C. (2020). FAG Journal of Health Prevalence of urinary tract infection on the elderly assisted by a preventive medical care program on Cascavel / PR Prevalência de infecção do trato urinário em idosos assistidos por um. 2 , $0-4$.

Fragoso dos Santos Fioravante, F., \& De Carvalho Queluci, G. (2017). Tecnologia educacional para a prevenção da infecção urinária nagravidez: estudo descritivo. Online Brazilian Journal of Nursing, 16(1), 28. https://doi.org/10.17665/1676-4285.20175447

Haddad, J. M., et al. (2018). Infecção do trato urinário. São Paulo: Federação Brasileira das Associações de Ginecologia e Obstetrícia (Febrasgo); 2018. (Protocolo Febrasgo - Ginecologia, nº 63/Comissão Nacional Especializada em Uroginecologia e Cirurgia Vaginal).

Lia, O., Steinberg, L., \& Paulo, S. (2015). Principais fatores de risco para a Infecção do Trato Urinário em gestantes na UBS Macuco Mauá/SP Especializando: Viviani de Araújo Sellan Gois Orientador: Lia Likier Steinberg.

Machado, A. D., Naumann, D. C., Ferrazza, M. H. S. H., Guevohlanian-Silva, A. T. B. Y., \& Weber, K. (2019). Prevalência de infecção urinária em um laboratório de análises clínicas da cidade de Jaraguá do Sul, SC, no ano de 2017. Revista Brasileira de Análises Clínicas, 51(3), 213-218. https://doi.org/10.21877/2448-3877.201900821

Oliveira, M. S., Trindade, G. do N. C., Machado, K. L. B., Santos, M. do C. M., \& Oliveira, E. H. de. (2021). Principais bactérias encontradas em uroculturas de pacientes com Infecções do Trato Urinário (ITU) e seu perfil de resistência frente aos antimicrobianos. Research, Society and Development, 10(7), e5310716161. https://doi.org/10.33448/rsd-v10i7.16161

Oliveira, S. M., et al. (2018). Infecção do trato urinário: estudo epidemiológico em prontuários laboratoriais. Journal Health NPEPS, 3 (1), 198-210.

Paula, M. L. A. de, Negri, M. M., Paula, C. L. A. de, Xavier, A. R., Kanaan, S., \& Weide, L. de C. C. (2016). Infecção do trato urinário em mulheres com vida 
Research, Society and Development, v. 10, n. 12, e402101220567, 2021

(CC BY 4.0) | ISSN 2525-3409 | DOI: http://dx.doi.org/10.33448/rsd-v10i12.20567

sexual ativa. J. Bras. Med, 37-41.

Paulo, P., Araújo, Y. B. De, Kelly, G., Leal, G., \& Júnior, S. (2021). Fatores de risco para infecções no trato urinário : revisão integrativa Risk factors for urinary tract infections : integrative review Factores de riesgo de infecciones del tracto urinario : revisión integral. 13(1), 1-8.

Santos, S. L. F., et al. (2018). Self-medication in High Risk Pregnant: Focus on Pharmaceutical Care. J Health Sci, 50 (4), 50-54.

Santos, M, J. S., et al. (2019). Etiologia e perfil de resistência bacteriana em uroculturas de pacientes atendidos em um hospital público de Macapá-Amapá, Brasil. Diagn Tratamento, 24 (4), 135-142.

Silva, P. P. A., et al. (2021). Fatores de risco par infecções no trato urinário: revisão integrativa. Revista Eletrônica Acervo Saúde, 13 (1), 1-8.

Simmering, J. E., et al. (2017). Clima mais quente como fator de risco para hospitalizações por infecções do trato urinário. Epidemiologia e Infecção, 146 (1), 386-393.

Tavares, V. B. (2017). Infecção Do Trato Urinário Na Gravidez Uma Revisão De Literatura. Caderno de Graduação - Ciências Biológicas e Da Saúde FACIPE, 2(3), 67.

Vaz, B. C., Silva, C. B. da, Machado, D. P. B., Bertelli, E. V. M., Lopes, J. G. F., Alves, K. E. S., \& Ferreira, V. Y. L. (2020). Educação em saúde na prevenção de infecção no trato urinário: relato de experiência / Health education in the prevention of urinary tract infection: experience report. Brazilian Journal of Health Review, 3(5), 13931-13940. https://doi.org/10.34119/bjhrv3n5-204 